\title{
Maladie de Charcot-Marie à hérédité liée au sexe. Rôle de la protéine connexine Cx32
}

Plusieurs formes de la maladie de Charcot-Marie (CM), dégénérescence du système nerveux périphérique, ont vu récemment leur lésion moléculaire identifiée $\left(\mathrm{m} / \mathrm{s} \quad n^{\circ} 8\right.$, vol. 7, p. 868 et $n^{\circ} 11$, vol. 9, p. 1273). Le type IA, dont le gène est en 17 pl1-12, est dû à une anomalie de la protéine périphérique de la myéline ou PMP22; le type IB, dont le gène est en lq22-23, à une anomalie de la protéine $\mathrm{P}_{0}$ de la myéline. Ces deux protéines sont également responsables d'une forme encore plus grave, la maladie de DéjerineSottas $\left(m / s n^{\circ} 1\right.$, vol. 10, p. 100). C'est le tour du type $\mathrm{IC}$, qui, contrairement aux deux formes précédentes, reconnaît une transmission récessive liée au sexe, de voir sa lésion moléculaire identifiée.

Depuis près de dix ans, des analyses de liaison et de recombinaison avaient fait localiser le gène de cette CMX sur la partie proximale du bras long de l'X, avant de la préciser en Xq13 [1], dans un intervalle limité entre deux marqueurs. Un gène codant pour un type de protéine appelé connexine, la connexine Cx32, étant connu pour être situé dans cette région [2], une équipe de Philadelphie (PA) et de Boston (MA, USA) décida de l'évaluer en tant que candidat [3]. La Cx32 est un membre de la famille des connexines. La fonction de ces protéines est de former des canaux jonctionnels (gap junctions) intercellulaires. Les cellules, bien que proches les unes des autres, laissent entre elles des intervalles de l'ordre d'un nanomètre. Les sous-unités des connexines sont incorporées dans des demi-canaux, ou connexons, qui, avec leurs contre-parties émanant des cellules voisines, forment des canaux complets, aboutissant à molécules allant jusqu'à $1 \mathrm{kDa}[4,5]$ (voir p. 218). Selon les tissus, les connexines possèdent des sous-unités différentes; il existerait 12 gènes distincts chez le rat [4]. Le clonage de leurs gènes a commencé en 1986 [6, 7]. Celui de la $\mathrm{Cx} 32$ code pour une séquence de 283 acides aminés (figure 1).

Bergoffen et al. [3] ont d'abord cherché en vain des remaniements du gène par Southern blot. Ils ont ensuite étudié son expression et l'ont trouvée dans plusieurs tissus, avec un maximum dans le foie et le nerf périphérique. Ils ont alors décidé de séquencer la partie codante chez des malades atteints de CMX. Sur huit familles à risque ils ont identifié sept anomalies: six mutations faux-sens et une insertion d'une base déplaçant la phase de lecture et aboutissant à une terminaison prématurée. Les autres, dont une existe dans deux familles apparemment indépendantes, sont localisées dans des segments considérés comme importants, en particulier dans un des segments transmembranaires (voir figure 1). Dans une famille, une mutation en position 142 semble nouvelle, puisqu'elle est absente chez les autres membres de cette famille. Il est remarquable que la Cx32, bien qu'abondante dans d'autres tissus comme le foie, n'influe en pathologie que sur le nerf. Il est probable que, dans d'autres tissus, des vicariances soient possibles, et que seul le nerf périphérique ne puisse en fournir.

Lorsqu'on regarde la distribution de la protéine Cx32 par immunohistochimie [3], on constate qu'elle est présente dans le nerf myélinisé au niveau des nouds de Ranvier et d'incisures, dites de SchmidtLanterman, et non le long des axones. Cette distribution diffère de celle des protéines PMP22 et $\mathrm{P}_{0}$, dont les anomalies sont responsables des types de maladie de Charcot-Marie à hérédité autosomique.

On possède ainsi désormais un tableau probablement complet des lésions moléculaires de la maladie de Charcot-Marie de type I, démyélinisante, alors que les recherches ne font que commencer pour le type II, axonal, dont on sait seulement que l'un de ses gènes est localisé en lp35-36.

J.C.D.

1. Bergoffen JA, Trofatter J, PericakVance MA, Haines JI., Chance PF, Fischbeck KH. L.inkage localization of X-linked Charcot-Marie-Tooth disease. Am J Hum Genet $1993 ; 52: 312-8$

2. Corcos IA, Lafrenière RG, Begy CR, LochCaruso R, Willard HF, Glover TW. Refined localization of human connexin 32 gene locus, GJB1, to Xq 13.1. Genomics $1992 ; 13$ : 479-80.

3. Bergoffen J, Schere SS, Wang S, Scott MO, Bone LJ, Paul DL, Chen K, Lensch MW, Chance PF, Fischbeck KH. Connexin mutations in X-linked Charcot-Marie-Tooth disease. Science 1993; 262: 2039-42.

4. Dermietzel R, Spray DC. Gap junctions in the brain: what type, how many and why? Trends Neurosci 1993; 16: 186-92.

5. Bennett MVL, Barrio LC, Bargiello TA, Spray DC, Moetzberg E, SaezJC. Gap junctions: new tools, new answers, new questions. Neuron 1991; 6: 305-12.

6. Paul DI. Molecular cloning of cDNA for rat liver gap junction protein. J Cell Biol 1986 ; 103: 123-34.

7. Kumar NM, Gilula NB. Cloning and characterization of human and rat liver cDNAs coding for a gap junction protein. J Cell Biol $1986 ; 103: 767-76$ 


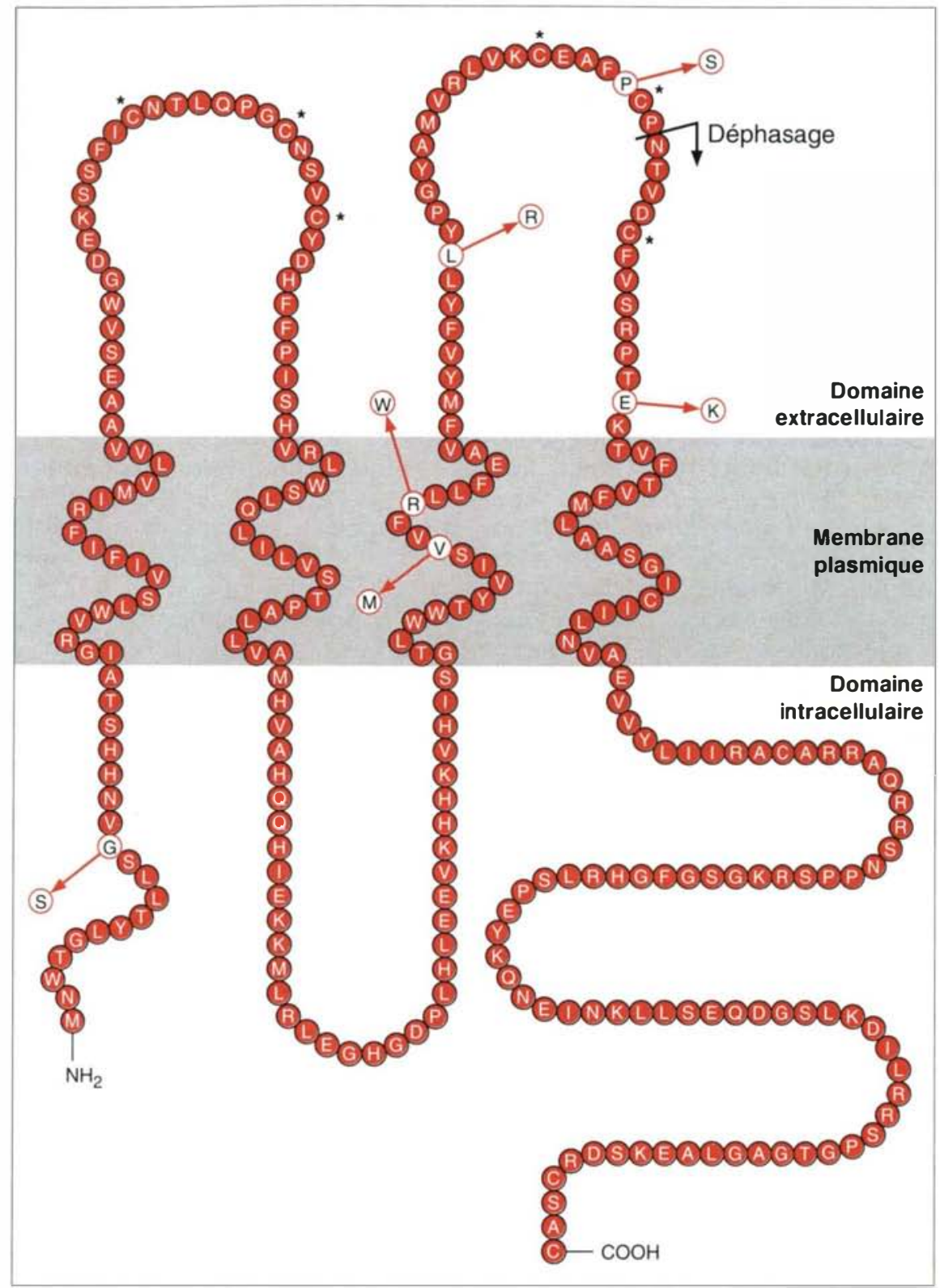

Figure 1. Représentation schématique de la connexine 32 (Cx32) d'après [3], montrant: dans la région intracellulaire les deux extrémités et une boucle; dans la région extracellulaire 2 boucles; et 4 segments transmembranaires. Les astérisques marquent les résidus cystéine. Les flèches indiquent les mutations: en notation à une lettre G12S, V139M, R142W, L156R, P172S, E186K, et le déphasage en 175.

Code à une lettre des acides aminés: $A$ : Ala; C:Cys; D: Asp; E: Glu; F: Phe; G: Gly; H: His; I: Ile; K: Lys; L: Leu; M: Met; N: Asn; P: Pro; $Q$ : Gln; R: Arg; S: Ser; T: Thr; V: Val; W: Trp; Y: Tyr.
La production de NO par l'endothélium vasculaire est-elle diminuée dans le diabète sucré insulinodépendant? Deux articles récents répondent positivement à cette question mais présentent des résultats contradictoires. La même technique a été utilisée : la réponse vasodilatatrice de l'avant-bras à la perfusion intra-artérielle d'analogues stables de l'acétylcholine, carbachol ou méthacholine, ou d'un inhibiteur de la synthèse de $\mathrm{NO}$, le L-NMMA. Johnstone et al. (Boston, MA, USA) montrent que l'action vasodilatatrice de la méthacholine est atténuée chez le diabétique, mais seulement à de fortes doses; cette réponse est inversement corrélée aux concentrations plasmatiques d'insuline [1]. Au contraire, pour Elliott et al. (Londres, GB), la réponse vasodilatatrice au carbachol est identique chez les diabétiques et les témoins mais l'effet vasoconstricteur du L-NMMA est accru chez les diabétiques, surtout chez ceux qui ont une microalbuminurie (qui est un facteur de risque pour le développement ultérieur de macroangiopathie et de néphropathie). En outre, la perfusion de L-NMMA réduit la réponse vasodilatatrice du carbachol chez les témoins et les diabétiques non microalbuminuriques, mais elle la supprime chez les diabétiques avec microalbuminurie (suggérant pour les auteurs que ces sujets ont une altération de la production de NO par l'endothélium) [2]. Il faut noter que les différences observées pourraient s'expliquer en partie par les différences suivantes : à Boston, les malades ont été prétraités par l'aspirine ; à Londres, une perfusion intraveineuse d'insuline est maintenue pendant l'étude alors que les diabétiques étaient sevrés d'insuline depuis 12 heures à Boston.

[1. Johnstone MT et al. Circulation $1993 ; 88: 2510-6$.

[2. Elliott TG et al. Clin Sci 1993; 85: 687-93.]
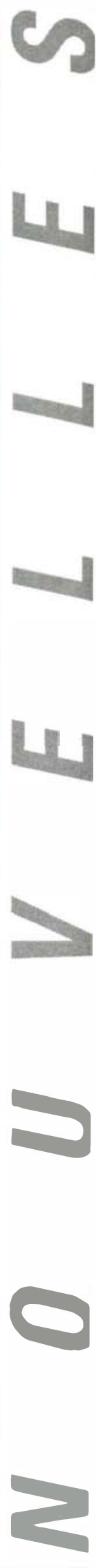